\title{
Deposition of thermally stable tungsten nitride thin films by reactive magnetron sputtering
}

\author{
A. H. Abdelhameed ${ }^{1}$, W. Jacob*
}

Max-Planck-Institut für Plasmaphysik, Boltzmannstr. 2, 85748 Garching, Germany

\begin{abstract}
Thermally stable tungsten nitride $\left(W_{2} N\right)$ thin films with thickness $\sim 0.9 \mu \mathrm{m}$ were successfully deposited on $S i$ and graphite substrates by reactive magnetron sputtering. The produced layers show no phase change or nitrogen release up to $1300 \mathrm{~K}$. These novel layers survived annealing at $1200 \mathrm{~K}$ for $50 \mathrm{hrs}$ without any measurable nitrogen loss or a phase transition from $W_{2} N$ to $b c c W$. X-ray diffraction, thermal desorption spectroscopy and Rutherford back scattering were utilized to characterize these layers and investigate the nitrogen release mechanism. The micro-structure of the layers was dominated by a grainy structure with porosity less than $16 \%$ and a strong texture in (200) orientation in contrast to the common (111) orientation. For annealing temperatures higher than $1200 \mathrm{~K}$ nitrogen release was observed, but this was attributed to a chemical reaction of $W_{2} N$ with the substrate material(s).
\end{abstract}

Keywords: Tungsten Nitride, Thermal Stability, Magnetron Sputtering, Decomposition, Nitrogen Release

Published in: $\quad$ Surface \& Coatings Technology 375, 701-707 (2019).

doi: $\quad$ 10.1016/j.surfcoat.2019.07.046

Submitted: $\quad 18.04 .2019$

Accepted: $\quad 22.07 .2019$

Available online: $\quad 31.07 .2019$

*Corresponding author. Tel.: +49 893299 2618; Fax: +49 89 3299 1504. E-mail address: Wolfgang.Jacob@ipp.mpg.de

${ }^{1}$ Current address: Max-Planck-Institut für Physik, Föhringer Ring 6, 80805, Munich, Germany. E-mail address: aab@mpp.mpg.de 


\section{Introduction}

Tungsten nitride $\left(W N_{x}\right)$ has attracted quite some interest in the past years due to its unique set of properties, such as excellent hardness, chemical stability, good thermal stability and high electrical and thermal conductivity [1, 2]. That made it a candidate for many potential applications such as $C u$ diffusion barrier into $S i$ in semiconductor applications [3, wear resistance protective coatings on mechanical components 4, catalyst for nitrogen monoxide dissociation and reduction with hydrogen [5], photoelectrochemical hydrogen production [6] or deuterium diffusion barrier for plasma-facing components in nuclear fusion devices [7]. $W N_{x}$ thin films have frequently been prepared by reactive magnetron sputtering 4, 7, 8, 9, 10, 11, 12, The formation of $W N_{x}$ is thermodynamically unfavorable, especially at atmospheric pressure. Consequently, most of the produced $W N_{x}$ was in the form of thin films via nonequilibrium processes. The produced layers possessed poor crystallinity, high residual stress and high porosity. Moreover, the reproducibility of such layers was very limited as many parameters can alter the overall properties.

Previous work focused extensively on studying the effects of deposition conditions on mechanical and electrical properties, micro-structure and chemical state configuration of the films. To date, very little is known about the thermal stability and the decomposition behavior of $W N_{x}$. Most applications of $W N_{x}$ often require not only good properties at room temperature, but also stability and functionality at elevated temperatures. Although thermodynamical predictions reported a thermal stability of $W_{2} N$ up to $2100 K$ [13, experimental investigations resulted in much lower $N_{2}$ release temperatures [10, 11, 12]. Shen et al. 12 reported $N_{2}$ release for their films starting at about $1090 \mathrm{~K}$ and being fully released at $1170 \mathrm{~K}$. $W: N$ films deposited by Gao et al. [1] showed an onset of $N_{2}$ desorption at 850 to $900 K$ and a release peak at 960 to $970 K$. Finally, Zhang et al. 10 reported an onset of $N_{2}$ release of their magnetron-sputter-deposited $W_{2} N$ layers at about $600 K$ continuously increasing and reaching a maximum release rate at about $1200 K$. For higher temperatures the release rate dropped strongly to very low values. Obviously the layer decomposed at $1200 \mathrm{~K}$. Furthermore, a dramatic degradation of the layers properties happened at temperatures of about $900 K$ [4]. So in summary, reported experimental values for the thermal stability of $W: N$ layer range from about 900 to $1200 \mathrm{~K}$. On the other hand, high uncertainties in quantifying the nitrogen amount in the deposited films made the study of the correlation of the films composition (stoichiometry), structural details, micro-structure evolution and decomposition behavior ambiguous. That is because of the low cross section of nitrogen in all spectroscopic methods, namely, Xray photoelectron spectroscopy (XPS) and parallel electron energy-loss spectroscopy (PEELS) .

In this work we show that fabrication of thermally sta- ble thin films by reactive magnetron sputtering is possible by optimizing the deposition parameters. The $W_{2} N$ layers produced in this work are long term stable for annealing at $1200 K$ for more than 50 hours. It will further be shown that the observed decomposition at higher temperatures is due to a reaction of the layer with the chosen substrate and not a property of the $W_{2} N$ layer itself.

\section{Experimental}

$W N_{x}$ layers were deposited on single crystal $10 \times 10 \mathrm{~mm}^{2}$ $S i(100)$ and $12 \times 15 \times 1.5 \mathrm{~mm}^{3}$ pyrolytic graphite substrates by reactive magnetron sputtering. Graphite substrates were cut in parallel to the graphite planes and were polished mechanically to achieve surface roughness $\sim 0.1 \mu \mathrm{m}$. The substrates were then cut and labeled using a laser cutting machine. In case of $S i$, initial cleaning of the substrates was done using a neutral soap solution followed by washing in a flow of deionized water for $2 \mathrm{~min}$. Soft wiping with dirt-free tissue was applied to fully clean and dry the surface. Finally all the substrates were rinsed vertically in high purity isopropanol in an ultrasonic bath for $10 \mathrm{~min}$ and were let to dry out, also vertically.

Depositions were carried out in a commercial Denton Vacuum Discovery-18 device. Prior to deposition, the system was pumped down to a pressure of $4.5 \times 10^{-5} \mathrm{~Pa}$ then a cold trap was filled with liquid nitrogen to minimize the water vapor pressure background. The substrate heater was turned on with a heating rate of $20 \mathrm{~K} / \mathrm{min}$. The samples were sputter-cleaned for $5 \mathrm{~min}$ by $A r$ plasma of pressure of $1.2 \mathrm{~Pa}$ created by applying $100 \mathrm{~W}$ RF power to the substrate holder which resulted in a DC self-bias voltage of $-500 \mathrm{~V}$.

DC current was applied to a tungsten target with purity of $99.98 \%$. The sputtering process was carried out in a mixture of $A r-N_{2}$ atmosphere. Prior to starting the deposition process the sputtering target was also sputtercleaned for $5 \mathrm{~min}$, with the same gas mixture as used for the deposition. We observed that the $W_{2} N$ phase could be produced using a wide range of the deposition parameters, however the layers had different properties. Therefore, a series of optimization of the deposition parameters was carried out (not discussed here) to produce the layers explained in this article. The best deposition parameters were found to be: $400 \mathrm{~W}$ from power-regulated DC power supply resulted in a target current of $819 \pm 5 \mathrm{~mA}$ and a sputtering voltage of $420 \pm 3 \mathrm{~V}$; the flow rates of $A r$ and $N_{2}$ were set by independent mass flow controllers to be 60 and $15 \mathrm{sccm}$, respectively ( $\mathrm{sccm}$ stands for cubic centimeter per minute at STP), yielding a total pressure of $2.0 \mathrm{~Pa}$. In the course of the deposition the pressure gradually increased to $2.4 \mathrm{~Pa}$. The distance between the cathode and the substrate holder was fixed at $10 \mathrm{~cm}$ (center to center) and the target had an inclination of $25^{\circ}$ since it is a multiple-cathode sputtering system. The deposition time to produce a thickness of $900 \pm 20 \mathrm{~nm}$ was $40 \mathrm{~min}$. 
Independent experiments have shown for layers deposited at low sample temperatures (no substrate heating) nitrogen release sets in at relatively low temperatures $(\sim 750 \mathrm{~K})$ [10]. We believe that this release is due to decomposition of some amorphous structures. Therefore, a sufficiently high substrate temperature was chosen for film deposition, aiming to reduce, or prevent, the formation of such amorphous structures with low decomposition temperatures. In the experiments reported here, the substrate temperature was set to be $\sim 800 K$. The temperature was measured in a separate identical deposition run using a thermocouple pressed against a $S i$ sample while the substrate holder was not rotating, since it was not possible to monitor the sample surface during the rotation of the substrate holder. Layers deposited under the here described conditions with a deposition temperature of $\sim 800 K$ will be called 'stable layers' in the remainder of this manuscript.

The crystallographic structure and preferred orientation were evaluated by X-ray diffraction (XRD) using a BraggBrentano diffractometer (Philips) in $\theta-2 \theta$ configuration. The X-ray source used the $C u K_{\alpha}$ line at $0.15418 \mathrm{~nm}$. In front of the source a $N i$ filter to remove $C u K_{\beta}$ and a $1.0 \mathrm{~mm}$ collimator were used. The diffractometer $\theta-2 \theta$ range was calibrated prior to the measurements with accuracy of $0.0001^{\circ}$. The samples were scanned in the range of $20-150^{\circ}$ with a step of $0.01^{\circ}$ and counting time of $10 \mathrm{~s}$ to enhance the signal-to-noise ratio. Texture measurements were done within $\chi$ range of $0-70^{\circ}$ with step of $5^{\circ}$ and $\phi$ range of $0-360^{\circ}$ with a step of $10^{\circ}$.

The surface morphology and the micro-structure were evaluated using a scanning electron microscope (SEM, Helios NanoLab 600 from FEI) combined with a focused ion beam (FIB) for cross-section imaging. The residual stress in the deposited films was evaluated by measuring the change of the substrate curvature due to film deposition using a Brukers Dektak stylus profilometer. Annealing up to $1300 K$ was performed in a full-molybdenum vacuum oven at a pressure of $2.7 \times 10^{-5} \mathrm{~Pa}$ with a heating and cooling rate of $1 \mathrm{~K} / \mathrm{min}$.

Thermal desorption spectroscopy measurements were carried out in a temperature programmed desorption device (explained elsewhere [14]). The sample is placed in a quartz tube inside an external, tubular oven. The oven heating ramp was $15 \mathrm{~K} / \mathrm{min}$. Sample temperature calibration was performed separately with a thermocouple attached to the surface of an identical $S i$ sample. The measured sample temperature showed a slight non-linearity up to $\sim 800 K$. Above $\sim 800 K$ the sample temperature was linear and about $\sim 20 K$ lower than the set oven temperature. The base pressure in the TDS system was better than $2 \times 10^{-8} \mathrm{~Pa}$. Prior to the measurements an identical ramp was performed without sample to desorb molecules which were adsorbed to the quartz tube walls during samples insertion. In addition, the background signal was then measured as a function of temperature and later subtracted from the measured data. The quadrupole mass spectrometer $(Q M S)$ was calibrated using two independent meth- ods for $D_{2}$ and $N_{2}$ gases, namely, by a LACO leak-bottle [15] ( $D_{2}$ only) and a self-made MINICAN-orifice system described in [16].

Rutherford backscattering spectroscopy (RBS) was carried out using the $3.0 \mathrm{MeV}$ tandem accelerator at IPP Garching, Germany. 3.0-5 $\mathrm{MeV}^{4} \mathrm{He}$ ion beam was used normal to the surface while the backscattering detector was at $165^{\circ}$. Quantifying the nitrogen content in the layers using RBS is of particular importance for the results presented here. However, quantifying light elements in a heavy matrix is not always straight forward. Because the cross section for detection of $N$ is much smaller than that for $W$, and because the $N$-related RBS signal for our layer system appears on top of the $S i$-RBS signal from the $S i$ substrate, the $N$ content cannot be determined directly from the $N$-RBS signal. But it is possible to determine the $N$ content in the $W: N$ layer from the decrease of the $W$-RBS signal compared with the $W$ signal of a pure $W$ layer or bulk $W$ if we can assume that $N$ is the only element in the $W: N$ layer that leads to the decrease of the $W$ signal.

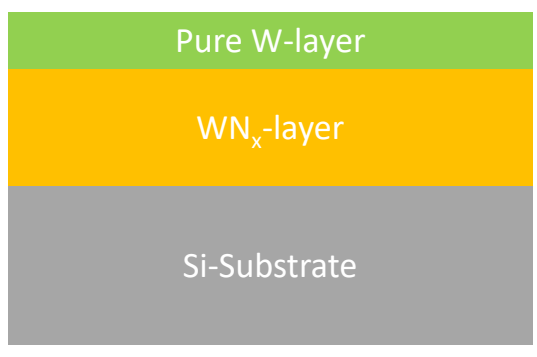

Figure 1: Schematics of the layer-system used to obtain accurate RBS measurements by depositing a thin pure-tungsten layer on top of $W N_{x}$.

This method depends on an accurate measure of the absolute $W$ signal height which varies on a day to day basis by about 5 to $10 \%$ due to uncertainties in the ion beam current measurement. Therefore, the such-determined $\mathrm{N}$ concentration in the layer has a relatively high uncertainty (of the order of 20-25\% of the determined value). Separate analyses by XPS had shown that the oxygen content in the $W: N$ and pure $\mathrm{W}$ layers which are deposited using our setup is negligible. Consequently, we can safely assume that the layers contained only nitrogen. The uncertainty of the $N$ concentration can be significantly reduced, if the W-RBS signal of a pure $\mathrm{W}$ layer can be measured simultaneously with the $W: N$ layer. Therefore, we deposited a pure tungsten layer (about $300 \mathrm{~nm}$ ) on top of the $W: N$ layer as schematically shown in Fig. 1. This pure tungsten layer was deposited in a separate deposition run prior to RBS analysis. The additional pure tungsten layer guarantees that the pure $W$ signal is measured for the identical conditions as the $W: N$ signal, thus decreasing the uncertainty for the determination of the $N$ concentration to about $2 \%$ (of the determined value). For quantitative 

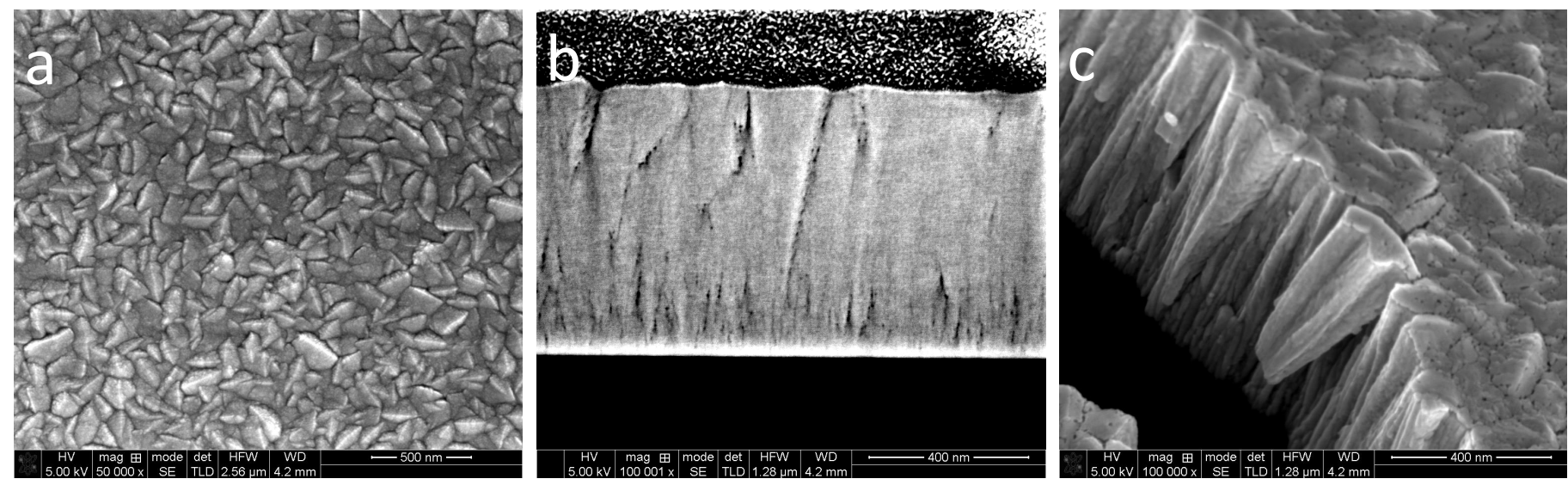

Figure 2: SEM images of stable layers: (a) the surface morphology shows the grainy structure, (b) cross-section made by FIB (vertical scale is $79 \%$ of the horizontal scale due to surface tilting), and (c) cross-section image of a crack that occurred after heat treatment shows the columnary structure more clearly.

analysis RBS spectra were evaluated using the SIMNRA code [17.

\section{Results}

\subsection{Film composition and micro-structure}

Fig. 2 shows SEM images of the surface morphology of the deposited layers. The structure is dominated by densely packed fibrous grains with structure size $>$ $100 \mathrm{~nm}$. The FIB cross-section image (Fig. 2b) shows the films thickness and a residual minor porosity mainly at the grain boundaries. A further cross-section image of a crack which has developed after heat treatment (Fig. 22) shows the columnar structure more clearly. Comparison of different surface and cross section images before and after heat treatment did not show a visible change of the columnar microstructure.

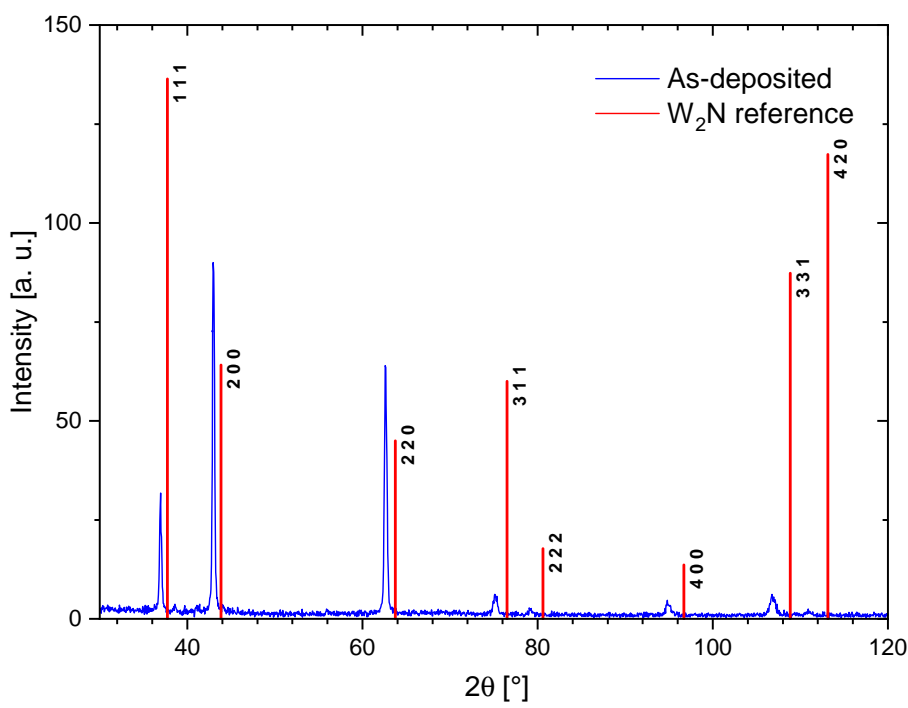

Figure 3: XRD $\theta-2 \theta$ scan of stable $W_{2} N$ indexed with the reference values (red lines) showing the peak positions and the relative intensities ICDD PDF-2 card 25-1257 18.
Fig. 3 shows the XRD pattern of the $W_{2} N$ layers compared with the reference peak-positions and relative intensities from ICDD PDF-2 card 25-1257 [18. The spectrum shows clearly higher intensities for the (200) and (220) orientation and a strong relative suppression of the (111) orientation compared with the reference spectrum. This means, the layers have a preferential (200) and (220) texture compared with the previously reported preferential (111) texture [9, 10]. In addition, the peaks show a shift towards lower angles (larger lattice plane distance) which indicates a possible tensile residual stress.

Some further information on the films texture is revealed by the pole figures presented in Fig. 4. Shown are pole figures for the three strongest peaks of the diffractogram presented in Fig. 3. namely the (111), (200) and (220) peak. The intensity color scale for the three subfigures is the same. The (200) and (220) peaks shown a fibre texture perpendicular to the sample surface. The mosaic spread of these two diffraction peaks is about $10^{\circ}$. A weak ring at about $45^{\circ}$ in the (200) pole figure is a diffraction spot that belongs to the planes with (220) orientation of the (200) grains. A corresponding structure in the (220) pole figure is not well visible. The (111) pole figure shows maximum intensity for a ring at about $27^{\circ}$. This is a diffraction spot that belongs to the grains with (111) orientation tilted by about $27^{\circ}$ to the surface normal. The corresponding (111)-plane of the preferential (220)-orientation on the surface texture should appear at $35^{\circ}$. The asymmetry in the intensity distribution for the (111) pole figure is probably due to a non-perfect mounting of the sample on the XRD sample holder.

Furthermore, we calculated the value of the residual stress in the film by measuring the change of the substrate curvature. The such-determined measured stress is found to be tensile and amounts to $\sim 0.35 G P a$. We believe that the residual stress in this film was mainly due to the thermal stress since the film was deposited at elevated temperatures and the measurements were done at room-temperature. The measured value of the residual 
$(111)$

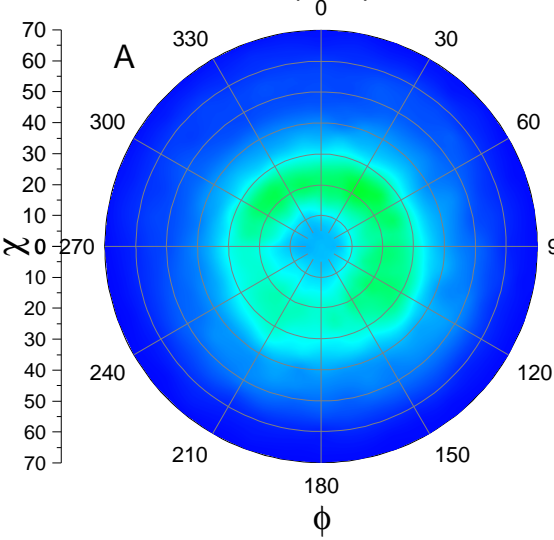

(200)

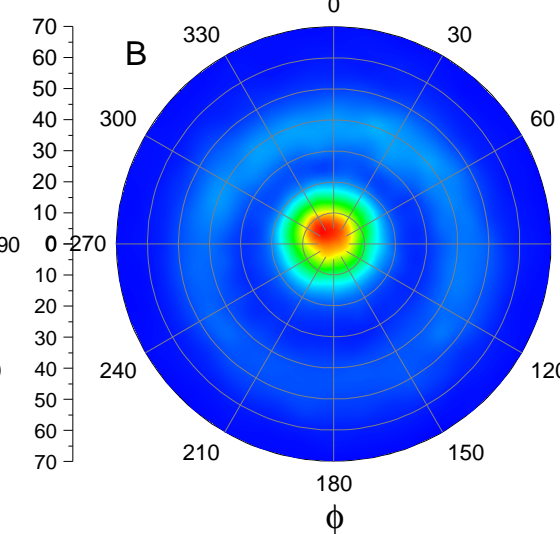

(220)

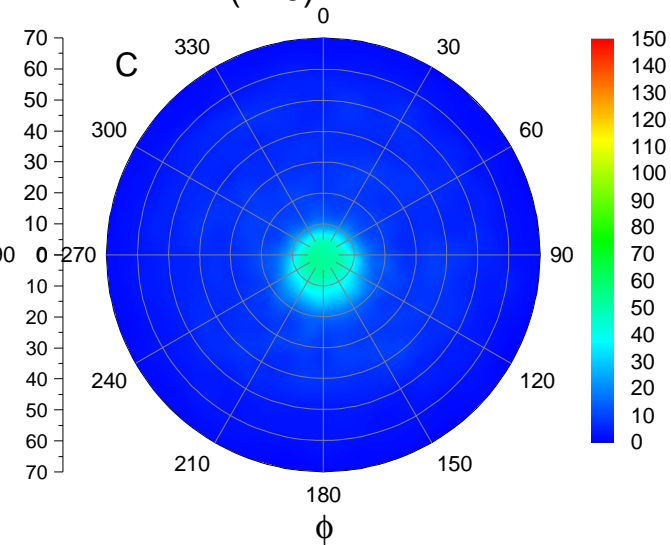

Figure 4: Pole figures showing the crystallographic texture of the layers. The individual figures present the intensities (color maps) of the first three peaks, (111), (200) and (220), as a function of the tilting angle $\chi$ and the rotation angle $\phi$.

stress was low and in opposite direction compared with the strain needed to shift the positions of the XRD peaks. The stress required to explain the observed peak shift that results in a change in the lattice parameter of $0.016 \AA$ is $\sim 9.0$ GPa calculated as sum of the principal stresses using 19 .

As described in Sec. 2, the $\mathrm{N}$ content in the layers was determined by the decrease of the $W$ signal of the $W: N$ layer in comparison with the $W$ signal of a pure $W$ layer. Fig. 5 shows the measured RBS spectrum of an stable $W_{2} N$ layer and the corresponding simulation result from the SIMNRA fit [17. The areal densities determined for this $W: N$ layer are $3.81 \times 10^{18} W$ atoms $/ \mathrm{m}^{2}$ and $2.51 \times 10^{18} \mathrm{~N}$ atoms $/ \mathrm{m}^{2}$. This results in an $N$ concentration of $40 \pm 2 \%$. The anticipated $\mathrm{N}$ concentration for stoichiometric $W_{2} N$ is $33 \%$. Therefore, the experimentally determined $N$ content is about 7 percent points too high which is significantly higher than the experimental uncertainty. We believe that this additional $N$ amount is bonded in amorphous $W: N$ phases with higher $\mathrm{N}$ concentration. With other words, that also means that about $17 \%$ of the total incorporated $\mathrm{N}$ amount in this $W: N$ layer is retained in a non crystalline phase.

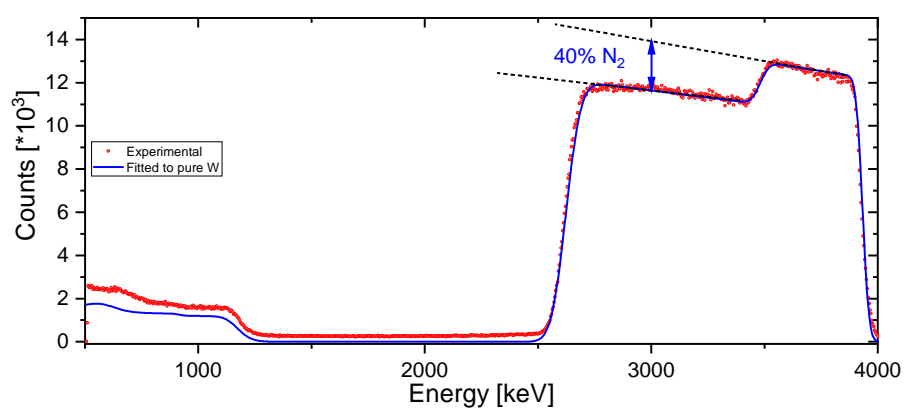

Figure 5: RBS spectrum of the $W_{2} N$ (red circles) coated with puretungsten layer. The blue line represents the SIMNRA simulation result [17].
We estimated the the porosity of the layers from the areal density determined by RBS and the thicknesses determined by SEM on a FIB cross section. The areal densities are given above and the SEM-determined layer thickness for this layer is $900 \pm 20 \mathrm{~nm}$. This results in an experimentally determined density of $14.8 \mathrm{~g} / \mathrm{cm}^{3}$. The theoretical value for the $W_{2} N$ density calculated from the lattice parameters [18] is $17.8 \mathrm{~g} / \mathrm{cm}^{3}$. The comparison of these two density values results in an average porosity of $\sim 16 \%$.

\subsection{Thermal stability and nitrogen-release characteristics}

Fig. 6 shows a comparison of $N_{2}$ desorption rates from two $W_{2} N$ layers produced in the frame of this work. As mentioned before, the $W_{2} N$ layer produced applying the deposition conditions described in Sec. 2 , in particular the high sample temperature of $800 K$ during deposition will be called 'stable layer'. The rational for that is the result presented in Fig. 6. The other layer will in the following be named 'common $W: N$ layer'. The deposition conditions for this common $W: N$ layer are: $N_{2} / A r$ flow ratio $=30: 40(\mathrm{sccm})$, total pressure of $2.0 \mathrm{~Pa}$, DC power of $300 W$ resulting in a bias voltage of $\sim 500 \mathrm{~V}$ and sample temperature of about $300 \mathrm{~K}$. In general, these deposition conditions are rather similar to reported work [10, 11] and to the conditions for deposition of the stable layer. The main difference for the deposition of the stable layer is the depositon temperature of $800 \mathrm{~K}$ instead of $300 \mathrm{~K}$. Although both layers are characterized to be $W_{2} N$ by XRD, they exhibited completely different thermal stability. The common $W: N$ layer starts to decompose at about 750 to $800 \mathrm{~K}$. The main release peak is at about $970 \mathrm{~K}$ and the $N_{2}$ release signal has not yet decreased to zero when the maximum temperature for the TDS run has been reached $(1323 \mathrm{~K})$.

Unfortunately, we were not able to measure a possible remaining amount of $N$ in the layers after TDS because 
the samples were either damaged by peeling off or or suffered from Si diffusion into the layer. However, the integral of the released $\mathrm{N}$ amount is in good agreement with the total $\mathrm{N}$ amount determined from the RBS analysis prior to TDS. We are, therefore, convinced that the vast majority of the $\mathrm{N}$ in the common $W: N$ layer was released during the TDS measurement. On the other hand, the stable layer shows almost no $N_{2}$ release in this temperature range. Only a very minor signal becomes apparent above about $1000 \mathrm{~K}$. The thermal stability of this stable layer is further investigated in the following.

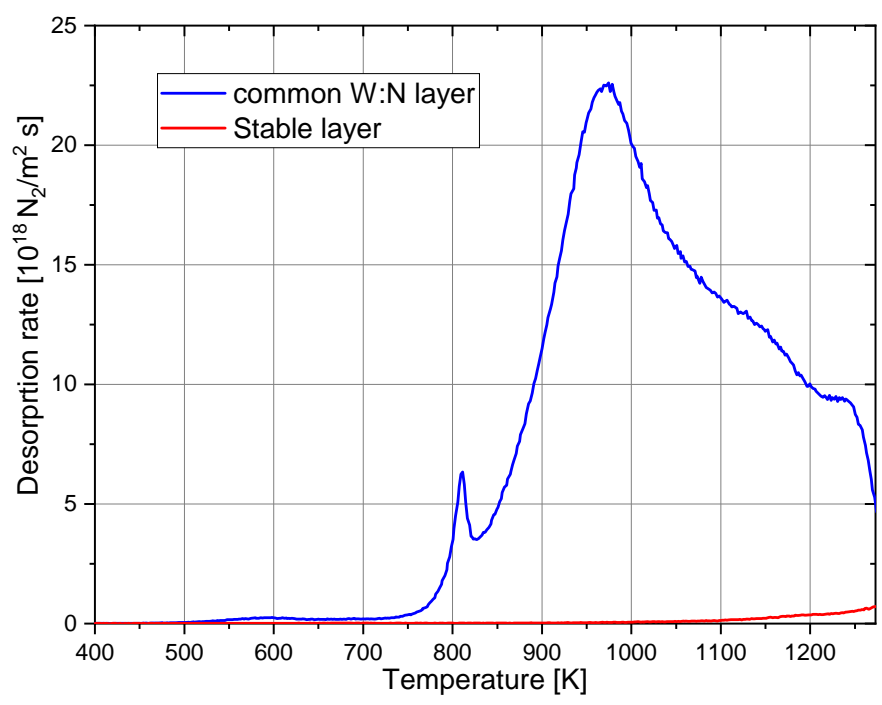

Figure 6: Comparison of the nitrogen release (recorded by the QMS 28 amu signal) behavior of the stable layer (red line) vs. a common $W: N$ layer (blue line) as a function of temperature. The released amount is negligible up to $1250 K$ (only $2.3 \%$ of the total amount) whereas almost all the nitrogen from the common $W: N$ layer is released.

Fig. 7 shows the nitrogen release behavior from an approximately $900 \mathrm{~nm}$ thick stable $W_{2} N$ layer deposited on $\mathrm{Si}$ as a function of time. Also shown (dashed blue line) is the oven temperature as a function of time. $N_{2}$ release sets in at about $1000 \mathrm{~K}$. The release rate increases steeply, resulting in a first peak at about $\sim 1250 \mathrm{~K}$ and then strongly decreases. This first desorption peak occurs slightly before the maximum temperature in this experiment was reached. After reaching $T_{\max }(=1300 \mathrm{~K})$, the sample was held at $T_{\max }$ for 3 hours. Surprisingly, during this holding time 2 additional, broad $N_{2}$ release peaks occurred. Two points are remarkable: First, the onset of $N_{2}$ release as well as the first desorption peak are well above previously reported [10, 11] $W: N$ decomposition temperatures. And second, integrating the $N_{2}$ amount released during the ramp up to $T_{\max }$ and comparing it with the total released amount we find that the former corresponds to $2.3 \%$ only. That means, the vast majority on $N_{2}$ released from the sample is released during the holding phase at $1300 \mathrm{~K}$.

As mentioned before in the discussion of the RBS results, the $N$ amount determined by RBS is about $17 \%$ higher than anticipated for a pure $W_{2} N$ phase. We assume that the small amount of $N$ released before reaching $T_{\max }$ in Fig. 7 is $\mathrm{N}$ released from this non-stoichiometric fraction of $N$.

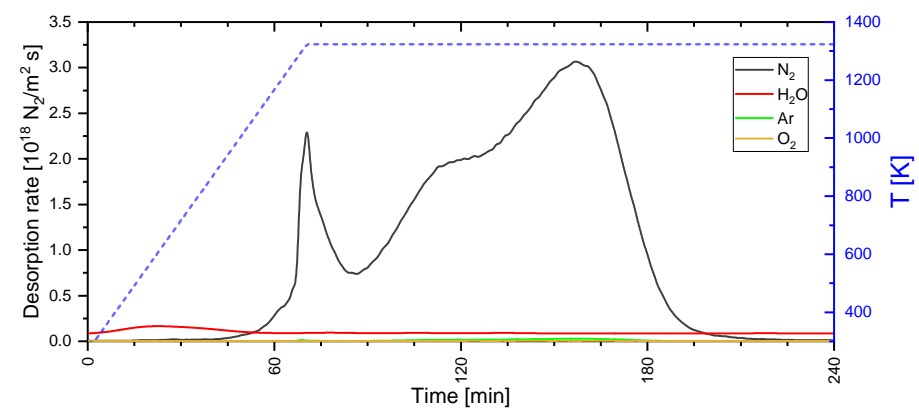

Figure 7: Desorption behavior of $\mathrm{H}, \mathrm{Ar}, \mathrm{H}_{2} \mathrm{O}$ and $\mathrm{O}_{2}$ from the $W_{2} N$ sample as a function of time recorded by QMS. One sharp peak visible on the left hand side represents the overstoichiometric nitrogen which is most probably retained in an amorphous phase. It is followed by two overlapping peaks which represent the main nitrogen-release. The dashed blue line shows the oven temperature. The sample temperature is about $20 \mathrm{~K}$ lower (see Sect. 2 .

In addition to the release of $N_{2}$, Fig. 7 shows the desorption behavior of other volatile species. We observed that the sum of all other species was less than $0.5 \%$ of the $N_{2}$ signal integral. Since all the nitrogen release happened at constant temperature in a relatively slow process of more than two hours, we do not consider that to be a thermal decomposition process.

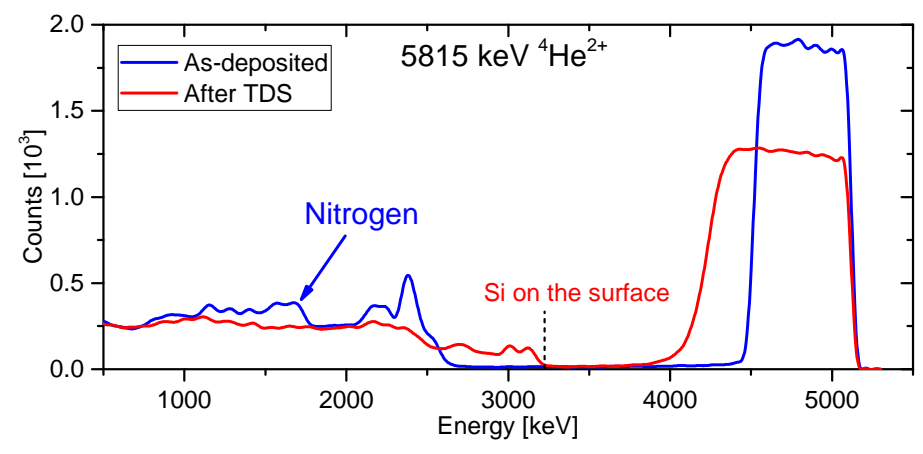

Figure 8: RBS spectrum of the as-deposited $W_{2} N$ layer in comparison with the RBS spectrum after after TDS to $1300 \mathrm{~K}$ and annealing at $1300 \mathrm{~K}$ for 3 hours as shown in Fig. 7

We suggest that the nitrogen release leading to the two broad peaks in Fig. 7(from about 90 to $180 \mathrm{~min}$ ) is caused by a diffusion of the substrate material $S i$ into the $W_{2} N$ layer and a corresponding replacement of $N$ by $S i$. Fig. 8 shows the RBS spectrum of the layer after the release of all nitrogen during TDS in comparison to the as-deposited spectrum by thermal treatment shown in Fig. 7 The RBS spectrum after annealing changed dramatically: $S i$ is now visible at the surface and simultaneously the $W$ peak became broader while the peak height became lower. Integration over the $W$ peak shows that the total $W$ amount remained constant. The change of the $W$ peak can be ex- 
plained by diffusion of $S i$ into the $W_{2} N$ layer. The weak $N$ peak which is visible in the region around $1500 \mathrm{keV}$ has disappeared after annealing. Together with the observation that the released $N$ amount during the experiment shown in Fig. 7 roughly agrees with the initial $N$ amount measured by RBS prior to annealing we conclude that the layer after annealing contains no significant amount of $N$. Assuming that the broadening of the $W$ peak is merely due to the diffusion of $S i$ into the $W_{2} N$ layer - implicitly this also means that $\mathrm{N}$ is completely replaced by $S i$ - the $W$ concentration in this layer is $34 \pm 0.5 \%$ o. That fits the stoichiometry of $\mathrm{WSi}_{2}$.

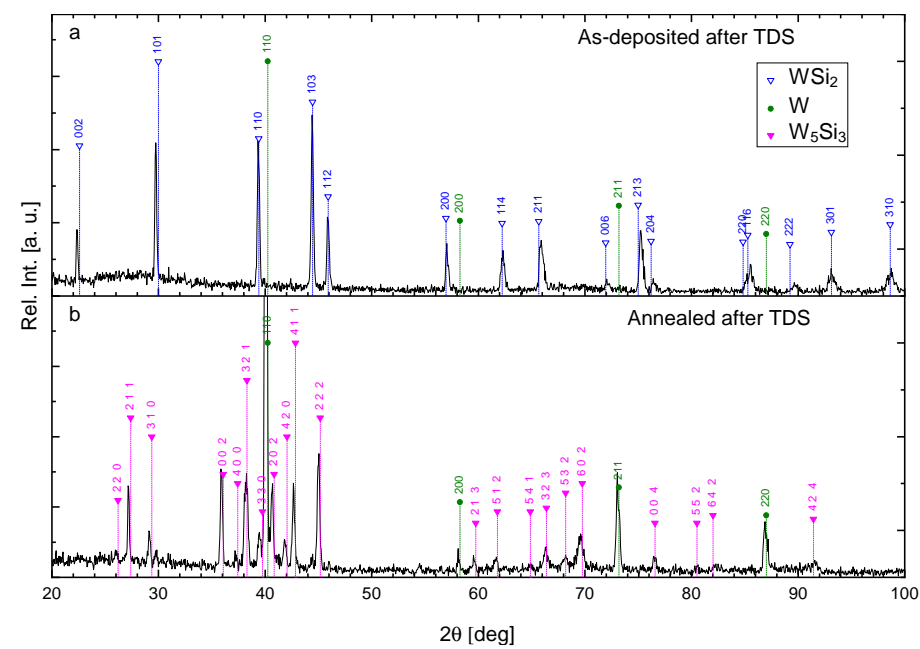

Figure 9: XRD $\theta-2 \theta$ scans of $W_{2} N$ after TDS and the release of the nitrogen from the as-deposited film (a) and from the annealed film at $1200 K$ for $50 h(\mathrm{~b})$.

Fig. 9 (top) shows that our XRD scan of the layer after performing TDS (after $N$ is released) fits exactly the $W \mathrm{Si}_{2}$ from ICDD PDF-2 card 81-165 [18. The scan is indexed with the peak positions of tungsten to show that no peaks from a pure tungsten phase appeared. Fig. 9 (bottom) shows the XRD pattern, after TDS, of the annealed sample at $1200 \mathrm{~K}$ for $50 \mathrm{~h}$. The XRD pattern fits the tungsten siliside phase $W_{5} S i_{3}$ ICDD PDF-2 card 81-165 [18.

The here presented RBS and XRD results consistently show that $S i$ diffuses into the $W_{2} N$ films and replaces $N$. The diffusion and replacement is finished after about $3 h$ annealing at $1300 \mathrm{~K}$. The $N$ from the film is released in form of $N_{2}$. This also means that the here observed $N$ release is not due to thermal decomposition of the $W_{2} N$ film but due to a chemical reaction of the diffused substrate material with the film. We can, therefore, speculate that the thermal stability of the film itself is even higher than $1300 K$. Nevertheless, is the here found thermal stability limit of the film-substrate system $300 K$ higher than the up to now highest reported stability limit.

A comparison was made between the $N_{2}$ (mass $28 \mathrm{amu}$ ) release signal from TDS measurements of identical layers deposited on $S i$ and graphite substrates. We observed that the nitrogen release from the layer deposited on the graphite substrate was much faster than from the layer deposited on $S i$. We attribute that to the difference in the diffusion coefficient of $S i$ and $C$ into $W$ [20].

\subsection{Annealing effects}

In literature, annealing of $W N_{x}$ showed contradicting results. Some researchers reported crystallization of the amorphous films at temperatures $\sim 873 K[9]$. However, that was not observed in the course of this study. Since we managed to minimize, to some extend, the amorphous fraction in the films the layers showed higher thermal stability. We performed annealing at $1200 K$ for $50 h$ in order to verify the stability of the layers beyond the reported values and to study the heat treatment effects in more detail. Our RBS analysis of the layers after annealing showed a negligible nitrogen loss $(\sim 0.6 \%)$. The results of our XRD measurements showed neither formation of pure $W$ phase nor a significant change of the crystallinity. However, this annealing had a clear effect on the peak position and we also observed a slight change on the film texture (see Fig. 10). After annealing, the peaks shifted towards the nominal peak position for $W_{2} N$ and the (200) peak gained strongly in intensity. The former is interpreted as a release of stress and the latter as a change in the film texture. We noticed that a slow heating and cooling ramp (1 $\mathrm{K} / \mathrm{min}$ in our case) was necessary to relieve the stress without damaging of the film (peeling) as a consequence of a thermal expansion.

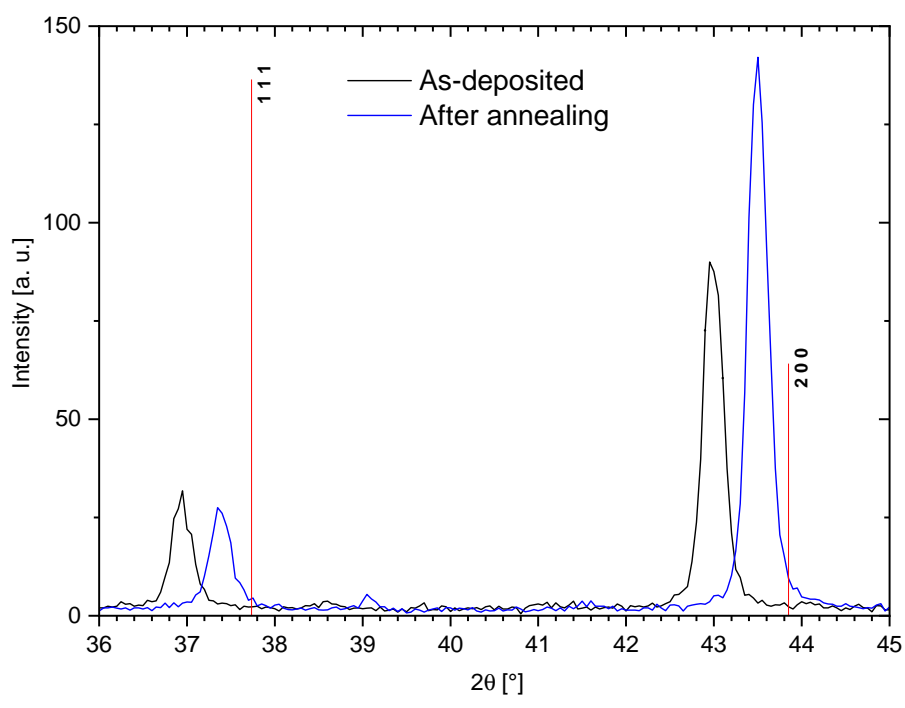

Figure 10: XRD $\theta-2 \theta$ scans of $W_{2} N$ layers indexed with the reference values. Red lines show the peak positions with relative intensities. Blue line is after annealing at $1200 \mathrm{~K}$ for $50 \mathrm{~h}$ in comparison with the as deposited layer (black line).

Fig. 11 shows RBS comparison of the layers after heat treatment. We did not notice any change on the layer homogeneity. 


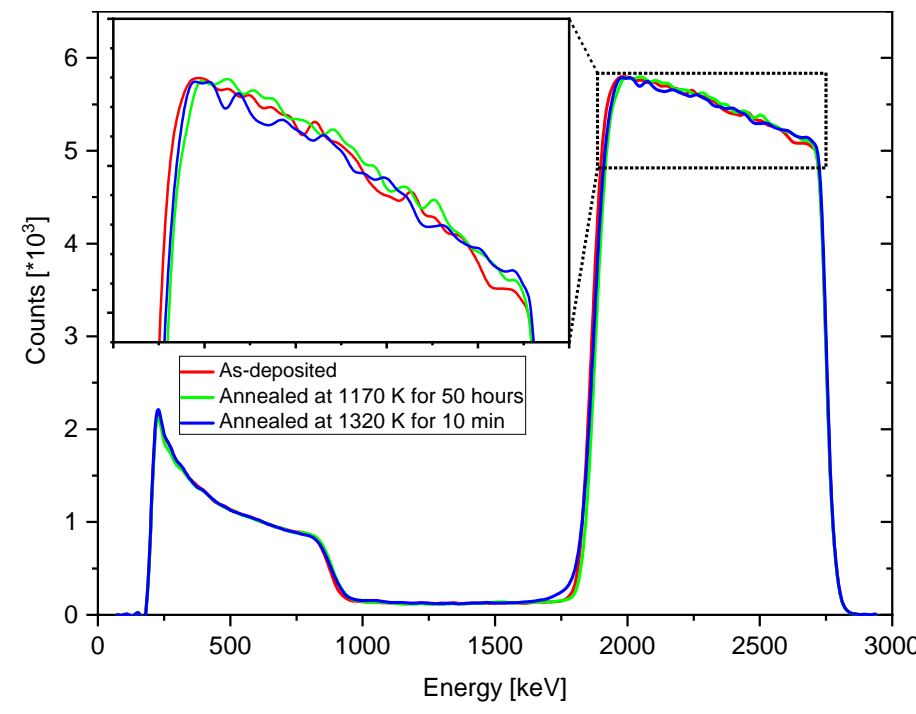

Figure 11: RBS spectra (smoothed raw data) of the $W_{2} N$ layer asdeposited (red line) and two annealed samples: at $1170 \mathrm{~K}$ for $50 \mathrm{~h}$ (green line) and at $1320 \mathrm{~K}$ for 10 minutes (blue line).

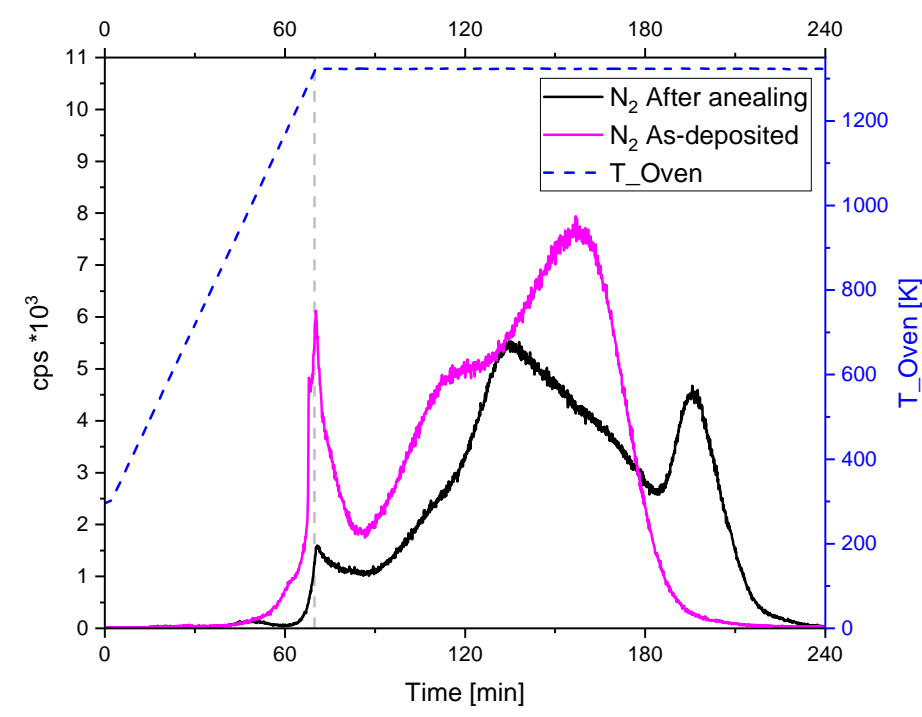

Figure 12: Desorption behaviors of nitrogen as a function of time recorded by QMS for the comparison of two $W_{2} N$ samples. Black line represents the desorption of the annealed sample in comparison with the desorption of the as-deposited sample.

In order to study the micro-structure changes due to annealing on the nitrogen release behavior, TDS, with the same conditions, was performed on an annealed film. Fig. 12 shows the desorption behavior of nitrogen from the annealed film compared with the desorption from the asdeposited layer. We observed a total delay of the main release of $\sim 30 \mathrm{~min}$, in addition to a different release behavior. The interpretation of such observations is not straightforward, however our suggestion is that diffusion of silicon into the film was slower. The observed shift of the XRD peaks (Fig. 10 towards higher angles means that the volume of $W_{2} N$ lattice was decreased. Together with the enhancement of the crystallinity through the min- imization of crystal defects, this might influence the diffusion of silicon into $W_{2} N$. Our XRD scan of the annealed film after TDS (Fig. 9 bottom) supports our claim. The XRD peaks show the formation of a tungsten silicide phase $W_{5} S_{3}$ which has a lower concentration of silicon. In addition, we also observed existence of $b c c \alpha-W$. That agrees with the phase diagram of $S i$-W system.

\section{Discussion}

The thermally stable $W_{2} N$ layers deposited in this work applying a deposition temperature of $800 K$ are dense layers with a columnar microstructure (see Fig. ??). XRD analysis reveals that the layers have a $W_{2} N$ phase with a preferential (200) and (220) texture (Fig. 3). RBS results are in a reasonable agreement with the predicted atomic concentrations for $W_{2} N$ stoichiometry which should yield $33 \%$ nitrogen. The nitrogen concentration found by RBS is about $40 \pm 2 \%$. This additional $N$ concentration of about 7 percent points corresponds to about $17 \%$ of the total $N$ amount in the film. The integral over the first release peak at about $1250 \mathrm{~K}$ shown in the TDS results in Fig. 7 corresponds to about $15 \%$ of the total released nitrogen amount. This value is in a good agreement with the overstoichiometric nitrogen amount measured by RBS. Taking further into account that the total released $N$ amount is in good agreement with the total $N$ amount measured by RBS, we, therefore, attribute this peak to the release of nitrogen which is either bonded at grain boundaries or in an amorphous phase.

The films exhibit superior thermal stability compared with films deposited at about $300 K$ (see Fig. 6) and reports from literature [10, 11, 12. They show no measurable $N$ release for temperature up to $1000 \mathrm{~K}$ and only a fraction of about $15 \%$ is released up to $1300 \mathrm{~K}$. The influence of long term annealing was investigated at $1200 \mathrm{~K}$ for up to $50 \mathrm{~h}$. The $N$ loss measured by RBS (0.6\%) was negligible (Fig. 11) and XRD did not show the appearance of another phase (Fig. 9). The only observable effect in the XRD spectrum was a slight peak shift and an increase of the (200) intensity (Fig. 10).

\section{Conclusion}

Thermally stable $W_{2} N$ thin films were deposited on $S i$ and $C$ substrates by reactive magnetron sputtering. It is possible to improve the layer properties, especially the thermal stability, by optimizing the deposition conditions. The important deposition parameter identified in our experiments is the sample temperature during deposition. At the here used deposition temperature of $800 \mathrm{~K}$ the $W_{2} N$ films possess superior thermal stability at least up to $1200 \mathrm{~K}$. A release of about $15 \%$ of the bonded nitrogen for temperatures after annealing to $1300 \mathrm{~K}$ was attributed to a small fraction of $\mathrm{N}$ bonded not in the $W_{2} N$ phase but possibly on grain boundaries or in an amorphous phase. 
The observed $N$ release for extended annealing at $1300 \mathrm{~K}$ was attributed to a chemical reaction of the here used substrate material (silicon and graphite) into the $W_{2} N$ layer which leads to a nitrogen release due to a chemical reaction of the substrate material with tungsten. This nourishes the expectation that the decomposition of the $W_{2} N$ phase occurs at even higher temperatures than $1300 \mathrm{~K}$.

\section{Acknowledgments}

The authors would like to thank a number of colleagues at IPP Garching for their continued support for performing the measurements. We thank Joachim Dorner and Michael Fußeder for support regarding the ion beam analysis, Thomas Dürbeck regarding the TDS measurements, Stefan Elgeti regarding the XRD measurements and interpretation, Martin Balden regarding SEM and FIB and Liang Gao regarding the magnetron sputter device.

\section{References}

[1] L. Toth, Transition metal carbides and nitrides, Elsevier, 2014.

[2] M. L. Addonizio, A. Castaldo, A. Antonaia, E. Gambale, L. Iemmo, Journal of Vacuum Science \& Technology A: Vacuum, Surfaces, and Films 30 (2012) 031506.

[3] J. S. Becker, R. G. Gordon, Applied Physics Letters 82 (2003) 2239-2241.

[4] T. Polcar, N. Parreira, A. Cavaleiro, Wear 265 (2008) 319 - 326. URL: http://www.sciencedirect.com/ science/article/pii/S0043164807006916 doi/https: //doi.org/10.1016/j.wear.2007.10.011

[5] C. Shi, X. Yang, A. Zhu, C. Au, Catalysis Today 9395 (2004) 819 - 826. URL: http://www.sciencedirect. com/science/article/pii/S0920586104003141 doi/https: //doi.org/10.1016/j.cattod.2004.06.102 selections from the presentations of the 3rd Asia-Pacific Congress on Catalysis.

[6] V. Chakrapani, J. Thangala, M. K. Sunkara, International Journal of Hydrogen Energy 34 (2009) 9050 - 9059. URL: http://wWw.sciencedirect.com/science/article/ pii/S0360319909014396 doi/https://doi.org/10.1016/j. ijhydene.2009.09.031

[7] L. Gao, W. Jacob, T. Schwarz-Selinger, A. Manhard, Journal of Nuclear Materials 451 (2014) 352 - 355. URL: http://www.sciencedirect.com/science/article/ pii/S002231151400244X doi/https://doi.org/10.1016/j. jnucmat.2014.04.029

[8] M. Wen, Q. Meng, W. Yu, W. Zheng, S. Mao, M. Hua, Surface and Coatings Technology 205 (2010) 1953 - 1961. URL: http://www.sciencedirect.com/science/article/ pii/S0257897210007310 doi/https://doi.org/10.1016/j. surfcoat.2010.08.082

[9] Y. Shen, Y. Mai, Surface and Coatings Technology 127 (2000) 238 - 245. URL: http://www.sciencedirect. com/science/article/pii/S0257897200006642 doi/https: //doi.org/10.1016/S0257-8972(00)00664-2

[10] X. Zhang, Y. Wu, B. Mu, L. Qiao, W. Li, J. Li, P. Wang, Journal of Nuclear Materials 485 (2017) $1-7$. URL: http://www.sciencedirect.com/science/article/ pii/S0022311516306523 doi/https://doi.org/10.1016/j. jnucmat.2016.12.009

[11] L. Gao, W. Jacob, G. Meisl, T. Schwarz-Selinger, T. Höschen, U. von Toussaint, T. Dürbeck, Nucl. Fusion 56 (2016) 016004.

[12] Y. Shen, Y. Mai, W. McBride, Q. Zhang, D. McKenzie, Thin Solid Films 372 (2000) 257 - 264. URL: http://www.sciencedirect.com/science/article/pii/
S0040609000010415

S0040-6090(00) 01041-5

doi https://doi.org/10.1016/

[13] B. Predel, N-W (Nitrogen-Tungsten), Springer Berlin Heidelberg, Berlin, Heidelberg, 1997, pp. 1-4. URL: https://doi. org/10.1007/10522884_2144 doi 10.1007/10522884_2144

[14] E. Salançon, T. Dürbeck, T. Schwarz-Selinger, F. Genoese, W. Jacob, Journal of Nuclear Materials 376 (2008) $160-168 . \quad$ URL: http://www.sciencedirect. com/science/article/pii/S0022311508001633 doi/https: //doi.org/10.1016/j.jnucmat.2008.02.070

[15] L. Technologies, Salt Lake City, Utah, USA (2018). URL: www . lacotech.com

[16] P. Wang, W. Jacob, L. Gao, T. Dürbeck, T. Schwarz-Selinger, Nuclear Instruments and Methods in Physics Research Section B: Beam Interactions with Materials and Atoms 300 (2013) 5461.

[17] M. Mayer, IPP Report 9/113, Max-Planck-Institut fuer Plasmaphysik, Garching, Germany, April (1997). URL: http://hdl. handle.net/11858/00-001M-0000-0027-6157-F

[18] International Centre for Diffraction Data PDF-2 2000 (Database), Technical Report, Newtown Square, PA, USA, 2000.

[19] M. E. Fitzpatrick, A. T. Fry, P. Holdway, F. A. Kandil, J. Shackleton, L. Suominen, Determination of Residual Stresses by Xray Diffraction, Measurement Good Practice Guide 52, National Physical Laboratory, Teddington, Middlesex, United Kingdom, TW11 0LW, 2005. ISSN 1744-3911.

[20] K. Schmid, J. Roth, Journal of Nuclear Materials 302 (2002) 96 - 103. URL: http://www.sciencedirect.com/ science/article/pii/S0022311502008073 doi/https: //doi.org/10.1016/S0022-3115(02)00807-3 